\title{
Repensando o fenômeno do envelhecimento na agenda política das cidades: a importância da promoção da mobilidade de pedestres
}

\author{
Rethinking ageing in the political agenda of cities: the importance of promoting pedestrian \\ mobility
}

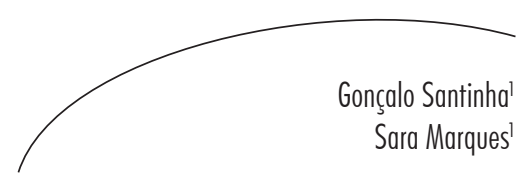

\section{Resumo}

As cidades confrontam-se atualmente com o duplo desafio de se assumirem cada vez mais enquanto polos de atração e aglutinadores de cidadãos e de concentrarem uma população tendencialmente em envelhecimento. Se é certo que várias orientações têm sido definidas no sentido de fomentar um novo olhar para a relação cidades/envelhecimento, não é menos verdade que a visão preconizada, compreensivelmente holística (abarcando temas como inclusão social, comunicação, emprego, habitação e transportes) carece de alguma especificidade e esclarecimento quanto à qualidade do espaço público e aos fatores que condicionam e geram maior mobilidade por parte dos cidadãos (em geral) e das pessoas idosas (em particular). Num contexto em que se salientam as questões de promoção de atratividade dos espaços enquanto mecanismos indutores de desenvolvimento econômico, por um lado, e em que se verifica a emergência de uma nova visão dos cuidados a prestar às pessoas idosas, designadamente ao apostar na sua permanência na comunidade (ageing in place), por outro, a temática em discussão revela-se de importância acrescida para a formulação de políticas públicas. Centrando-se essencialmente no domínio da organização territorial dos serviços e da mobilidade de pedestres, este texto procura contribuir para o debate, sublinhando sua importância, analisando a bibliografia existente e identificando as principais áreas de intervenção a considerar na construção de uma comunidade que proporcione às pessoas idosas condições que permitam otimizar as capacidades individuais e manter uma qualidade de vida saudável.

\section{Abstract}

Cities are now facing a double challenge: not only urban population is growing but it is also getting older. Though several guidelines have been developed in order to look at this cities/ageing nexus through a new perspective, the overall view, holistic by nature (involving issues such as social inclusion, communication, employment, housing and transports), lacks some specificity and clarification in what concerns the quality of public space and the aspects that prevent and generate mobility amongst

Palavras-chave:

Envelhecimento. Cidade Saudável. Mobilidade de Pedestres. Políticas Públicas. Qualidade de Vida.

Departamento de Ciências Sociais, Políticas e do Território. Universidade de Aveiro. Aveiro, Portugal. 
the elderly. In this context of promoting attractive spaces as economic development engines, on the one hand, and striving to maintain older people in their community (ageing in place), on the other, the issue at stake is of added value for policy making and delivery. By focusing mainly in the subject of service's spatial location and pedestrian mobility, this paper develops an understanding of this subject by synthesising the evidence and providing an interdisciplinary perspective that potentially benefits public policy debates regarding the development of an elderly friend community capable of providing conditions to optimise individual capacities and maintain a healthy quality of life amongst older people.
Key words: Aging. Healthy City. Pedestrian Mobility. Public Policies. Quality of Life.

\section{INTRODUÇÃO}

O envelhecimento da população e uma sociedade cada vez mais urbana em que as cidades assumem crescente papel de protagonismo são duas realidades que marcam o século XXI. Os países desenvolvidos, onde o processo de envelhecimento assume maior expressão, ainda se encontram em fase de adaptação às alterações que o fenômeno acarreta. Se nos centrarmos na imagem social da pessoa idosa, no contexto das características da sociedade contemporânea, observamos a desvalorização de seu papel, não só no que se refere à perda de potencialidades e produtividade, mas também por motivos associados à ideia de que esta representa um encargo social. Neste sentido, o envelhecimento da população tem merecido a preocupação de diversos organismos mundiais, nomeadamente a Organização de Cooperação e Desenvolvimento Econômico (OCDE), a Comissão Europeia (CE), a Organização das Nações Unidas (ONU) e a Organização Mundial da Saúde (OMS), que, sobre esta questão, avançaram com um conjunto de preocupações e recomendações na sua agenda e nos relatórios publicados.

Simultaneamente, a sociedade está se tornando cada vez mais urbana: a proporção global de população mundial urbana aumentou de 13\% em 1900 para 29\% em 1950, atingindo $49 \%$ em 2005. ${ }^{1}$ De acordo com as previsões demográficas, ${ }^{1} 60 \%$ da população mundial viverão nas cidades até 2030 . Assim, as cidades são, do ponto de vista da política pública, consideradas cada vez mais como um meio de oportunidade de desenvolvimento, tanto no nível da criação de condições de desenvolvimento econômico, como na procura de afirmação enquanto espaços de inclusão.

Cientes das alterações que a combinação do binômio população envelhecida/população urbana representa para a organização da cidade, autoridades locais e órgãos de representação internacional iniciaram um conjunto de trabalhos com vistas a perceber e antecipar áreas passíveis de intervenção, visando melhorar a qualidade de vida, tanto no caso particular das pessoas idosas, como para a população em geral. O exemplo recorrentemente citado nos meios acadêmicos e políticos consiste no trabalho colaborativo iniciado em 2005 pela OMS, com 35 cidades de todos os continentes, com o objetivo de identificar as principais características de um ambiente amigo da pessoa idosa. ${ }^{2}$

Este texto procura contribuir para o debate em torno desta relação cidades/envelhecimento, centrando-se no domínio da qualidade do espaço público numa ótica de políticas públicas, em que a organização territorial dos serviços e a mobilidade de pedestres se assumem como fatores determinantes para a qualidade de vida das pessoas idosas. Uma temática atual que se encontra na agenda política internacional, mas que necessita de maior visibilidade.

\section{RESPOSTAS DE APOIO ÀS PESSOAS IDOSAS: DA PERSPECTIVA TRADICIONAL À EMERGENTE}

À velhice associa-se frequentemente o conceito de perda, pela diminuição de capacidades do indivíduo, quando se valorizam 
os fatores negativos desta etapa da vida, tal como a perda do papel profissional e as alterações na estrutura familiar e na comunidade. ${ }^{2} \mathrm{~A}$ velhice representa também uma fase em que os indivíduos são mais vulneráveis e, como tal, mais expostos a situações de pobreza e outras debilidades, carecendo inúmeras vezes do apoio direto ou indireto do Estado. Contrariando este padrão global de associar ao envelhecimento características menos positivas, surgiu em 2002, na Conferência Mundial da OMS sobre o Envelhecimento, o conceito de Envelhecimento Ativo. A palavra "ativo" refere-se à participação contínua nas questões sociais, econômicas e culturais, espirituais e civis, e não somente à capacidade de estar fisicamente ativo ou integrar o mercado de trabalho. ${ }^{3} \mathrm{O}$ envelhecimento ativo define-se, pois, como um processo de otimização de oportunidades para a saúde, participação e segurança, no sentido de aumentar a qualidade de vida durante o envelhecimento. ${ }^{4}$

Adotar este ponto de vista significa reconhecer que as pessoas idosas desempenham novos papéis, permanecendo independentes durante mais tempo, trabalhando informalmente (desempenhando tarefas domésticas e outros trabalhos em pequena escala) ou exercendo atividades de voluntariado, manifestando simultaneamente uma procura diferenciada de serviços em consequência de níveis mais elevados de educação. Se é certo que com o aumento da população envelhecida verificou-se incremento significativo da procura de respostas sociais e econômicas em todo o mundo, ${ }^{5}$ não é menos verdade que a emergência deste novo olhar para as pessoas idosas reforçou a necessidade de se efetuar uma reflexão mais abrangente sobre o modo como as políticas sociais as têm encarado e sua contribuição para a promoção de um envelhecimento ativo.

Abordar esta questão implica repensar o próprio papel das pessoas idosas nas comunidades. Segundo Gilroy \& Castle, ${ }^{6}$ o lugar das pessoas idosas na comunidade tem sido negligenciado, pois não há toda a infraestrutura necessária à sua plena participação: quando se pensa nas infraestruturas que tendem a ter maior impacto sobre a vida das pessoas idosas, a habitação, os serviços sociais e os serviços de saúde surgem como principais elementos. E embora essas questões sejam relevantes, outras há que importa igualmente atender, mas às quais se tende a atribuir papel de ator secundário.

Neste contexto, é importante pensar na localização dos equipamentos e serviços de apoio às pessoas idosas, contrariando o terminal decline model, ${ }^{7}$ que considera a pessoa idosa como vítima de um processo de dependência e de perda irreversível. De fato, começam a surgir com maior frequência trabalhos de pesquisa com o objetivo de documentar as características da comunidade que as pessoas mais idosas identificam como as mais importantes para seu bem-estar. ${ }^{8-10}$ Esses trabalhos têm por base a realização de inquéritos, entrevistas ou sessões de trabalho, com pessoas que tenham mais de 55 anos de idade, que são convidadas a manifestar seu nível de satisfação com alguns elementos da cidade, nomeadamente, transportes, espaços exteriores e edifícios, respeito e habitação, participação cívica e emprego, apoio comunitário e serviços de saúde.

Apesar de se verificar atualmente um aumento da bibliografia que discorre sobre a relação entre o desenho da comunidade e a qualidade de vida (como sejam o novo urbanismo, as cidades saudáveis ou as comunidades pedestres), não existe pesquisa suficientemente clara que auxilie os decisores políticos na criação de uma comunidade amiga das pessoas idosas, na medida em que nenhum desses conceitos atende especificamente às necessidades que a população idosa apresenta. Os trabalhos acadêmicos desenvolvidos ${ }^{8,11,12}$ e as iniciativas políticas associadas a conceitos como streets for life, lifetime homes, sustainable communities* ou mesmo as já mencionadas cidades amigas das pessoas idosas, constituem exceções neste panorama e uma boa base de partida para aprofundar a questão.

\footnotetext{
* Esses conceitos emergiram com o intuito de acautelar um conjunto de princípios que propiciam a participação e integração dos indivíduos, para que durante todo o seu ciclo de vida desfrutem de uma sensação de bem-estar e de conforto na comunidade onde decidem viver. ${ }^{15}$
} 
Embora as abordagens sejam diversas e as temáticas estudadas abrangentes, no que respeita à qualidade do espaço público há dois componentes comuns aos trabalhos e às iniciativas mencionadas: a) a localização dos serviços, sua relação com a envolvente, disponibilidade, características e diversidade; b) a importância que a mobilidade de pedestres assume enquanto fator promotor ou dissuasor de apropriação do espaço e realização de atividades vitais para os cidadãos.

\section{A ORGANIZAÇÃO TERRITORIAL DOS SERVIÇOS E A MOBILIDADE DE PEDESTRES COMO ATRIBUTOS DA QUALIDADE DO ESPAÇO PÚBLICO}

São vários os autores que se têm debruçado sobre a importância da organização e disposição territorial dos serviços e equipamentos para um melhor usufruto dos mesmos por parte dos cidadãos. Os trabalhos desenvolvidos por Carstens $^{13}$ e Tolley $^{14}$ constituem marcos de referência na literatura, apontando geralmente uma distância de $400 \mathrm{~m}$ para os serviços de necessidades básicas e de $800 \mathrm{~m}$ para outros serviços. No primeiro caso, os cidadãos demorariam cerca de cinco minutos a percorrer a distância, enquanto que no segundo seriam precisos dez minutos.

Independentemente do tipo de serviços e equipamentos que devem ser considerados primários ou secundários em termos de acesso, importa aqui sublinhar o fato de ser crucial programar e localizar conjuntamente estes serviços e equipamentos numa determinada área de influência (com ganhos ao nível de acessibilidade e de economias de escala). Mais recentemente, ${ }^{15}$ adotou-se uma postura crítica em relação a esses indicadores, argumentando que estes critérios não tiveram em consideração as características das pessoas idosas com repercussões ao nível da mobilidade, independentemente do próprio grupo também não ser homogêneo. ${ }^{16}$ De acordo com o estudo efetuado, os autores concluem que, para um idoso, os tempos duplicam para dez e vinte minutos, respectivamente. Atender a estas especificidades torna-se importante, não só para garantir alternativas no acesso aos polos de serviços, nomeadamente pela disponibilidade de transportes coletivos, mas também para promover espaços de circulação e de permanência e mobiliário urbano adequados.

A distância, contudo, não é o único fator preponderante quando se analisa a localização de um equipamento: o padrão de ocupação e uso do solo e as condições de mobilidade de pedestres constituem atributos que assumem, neste contexto, particular importância. Em qualquer trajeto há um componente de deslocamento das pessoas que se pode realizar no início, durante ou na parte final deste. E a maior ou menor facilidade de deslocamento está relacionada com a forma como o ambiente construído apoia e incentiva o caminhar, fornecendo conforto e segurança aos pedestres, oferecendo variados destinos num período de tempo e esforço razoáveis. ${ }^{17}$ Relacionase, igualmente, com os fatores que induzem as pessoas a se deslocarem. ${ }^{18}$ Por outras palavras, a simples existência de espaços desprovidos de constrangimentos físicos à mobilidade é condição necessária mas não suficiente para induzir o deslocamento dos cidadãos.

Apesar de cada vez mais estudos se debruçarem sobre esta questão - realçando a importância de se adotar uma visão mais abrangente da mobilidade de pedestres -, poucos são os que apresentam uma metodologia que permita desenhar os espaços e formular políticas promotoras deste modo, focando designadamente as pessoas idosas. Como consequência, a maioria das políticas locais tende a centrar-se na remoção de barreiras arquitetônicas e urbanísticas enquanto mecanismo de melhoria dos espaços públicos e de indução da mobilidade de pedestres. ${ }^{19}$ A título de exemplo, recentemente começaram a ser desenvolvidas e aplicadas (essencialmente nos EUA) ferramentas analíticas de medição da qualidade dos espaços pedonais em muito baseadas nesta ótica. ${ }^{20}$ Embora medidas desta natureza sejam relevantes, esta visão se apresenta redutora face à importância da questão na qualidade de vida das pessoas idosas. 
CRITÉRIOS A SEREM CONSIDERADOS NA FORMULAÇÃO DE POLIITICAS PROMOTORAS DA MOBILIDADE DE PEDESTRES: SISTEMATIZANDO IDEIAS

Os estudos que se têm debruçado sobre esta problemática, embora apresentando dimensões analíticas distintas, tendem a realçar quer as características físicas do ambiente construído, quer os fatores sociais e as redes de relações que são induzidas e que ao ambiente construído se associam, questões cruciais na formulação de políticas desta natureza, com especial relevância no caso das pessoas idosas. ${ }^{21-24}$ Neste sentido, foi feita uma análise temática e interpretativa de sete trabalhos recentes de referência na literatura, que apresentam uma visão mais abrangente sobre a mobilidade de pedestres. ${ }^{15,17,18,20,22,23,25}$

Embora esses estudos apresentem critérios distintos em número e designação, diversas similaridades foram detectadas quanto à natureza e conteúdo desses mesmos critérios, verificando-se, por isso mesmo, uma interessante complementaridade no duplo ponto de vista conceitual e operacional. A análise conjunta da informação recolhida permitiu construir um quadro-síntese analítico, útil enquanto base conceitual para intervenções no âmbito da promoção da mobilidade de pedestres, no qual se apresentam os critérios a adotar, seu significado e as dimensões-chave a considerar na formulação de políticas públicas em que o fenômeno do envelhecimento ocupa papel relevante (quadro 1). Os critérios definidos - conectividade, conveniência, convivência, legibilidade e conforto - seguem a nomenclatura do trabalho pioneiro de Mayor of London, ${ }^{22}$ na medida em que sua diferenciação é a que se apresenta mais clara, sem ser demasiado exaustiva em número.**

\footnotetext{
** Ainda que descritos de forma bastante sucinta, e por isso mesmo passíveis de interpretações várias, os critérios definidos em Mayor of London ${ }^{22}$ foram designados por 5 C's: Connected, Convivial, Conspicuous, Comfortable, Convenient.
} 
Quadro 1 - Quadro-síntese analítico de critérios de intervenção para a promoção da mobilidade de pedestres.

\begin{tabular}{|c|c|c|}
\hline Critério & Definição & Dimensões-chave \\
\hline : & $\begin{array}{l}\text { Rede de percursos contínuos, sem } \\
\text { barreiras, e que asseguram uma boa } \\
\text { ligação entre os principais polos } \\
\text { de atração, bem como à rede de } \\
\text { transportes coletivos }\end{array}$ & $\begin{array}{l}\text { - Densidade e dimensão de quarteirões e } \\
\text { número de intersecções } \\
\text { - Barreiras naturais e impactos topográficos } \\
\text { - Localização de serviços e equipamentos } \\
\text { - Articulação entre modos de transportes }\end{array}$ \\
\hline 过 & $\begin{array}{l}\text { Rede de pedestres o mais direta } \\
\text { possível, combinando os caminhos } \\
\text { pedonais, os passeios, as vias mistas } \\
\text { com tráfego moderado, as passadeiras } \\
\text { ou passagens subterrâneas, bem como } \\
\text { as zonas para pedestres }\end{array}$ & $\begin{array}{l}\text { - Distância até serviços e equipamentos } \\
\text { - Trajetos alternativos (áreas de maior versus } \\
\text { menor tráfego) } \\
\text { - Atravessamentos (de nível, subterrâneos, aéreos) } \\
\text { - Largura útil (espaço e função) }\end{array}$ \\
\hline ن류. & $\begin{array}{l}\text { Deslocamento pleno e agradável sem } \\
\text { quaisquer restrições, proporcionando } \\
\text { uma sensação de calma e acolhimento, } \\
\text { num espaço livre de barreiras, } \\
\text { pavimentos de elevada qualidade, } \\
\text { desenho atraente, mobiliário dedicado } \\
\text { ao descanso e abrigos }\end{array}$ & $\begin{array}{l}\text { - Barreiras arquitetônicas e urbanísticas } \\
\text { - Fluxos de pedestres } \\
\text { - Iluminação e mobiliário urbano } \\
\text { - Estruturas de descanso }\end{array}$ \\
\hline 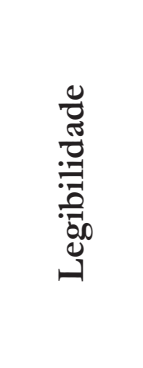 & $\begin{array}{l}\text { Percursos facilmente percetíveis, fáceis } \\
\text { de encontrar e de seguir }\end{array}$ & $\begin{array}{l}\text { - Informação (ex.: placas informativas, balcões } \\
\text { de atendimento) } \\
\text { - Sinalização (tendo em conta linguagem, cor e } \\
\text { contrastes) } \\
\text { - Diversidade arquitetônica, de materiais e cores } \\
\text { - Elementos marcantes e diferenciadores (ex.: } \\
\text { monumentos, praças, parques) }\end{array}$ \\
\hline 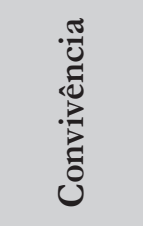 & $\begin{array}{l}\text { Atratividade dos espaços, variedade } \\
\text { e riqueza arquitetônica e cultural e } \\
\text { interação social }\end{array}$ & $\begin{array}{l}\text { - Interesse visual do ambiente construído } \\
\text { - Segurança } \\
\text { - Relações sociais } \\
\text { - Fatores de atração (ex.: cultura, desporto) }\end{array}$ \\
\hline
\end{tabular}




\section{CONSIDERAÇÕES FINAIS}

O texto apresentado deve ser assumido como um exercício de reflexão e atualização do debate em torno da complexa relação cidades/ envelhecimento. Se é certo que a ideia de uma população tendencialmente envelhecida não configura, à primeira vista, um cenário animador, fomentando inclusive uma imagem negativa do papel da pessoa idosa na sociedade, não é menos verdade que a percepção deste fenômeno numa ótica de oportunidade para os territórios não só é desejável, como é possível.

Transformar este desafio em oportunidade depende dos mecanismos que as cidades e seus protagonistas acionarem e articularem em consonância com as diretrizes que vêm sendo definidas por instâncias nacionais e internacionais e com o apoio da comunidade acadêmica. Uma vez reconhecido este princípio, torna-se necessário identificar com maior rigor quais os critérios técnico-científicos a adotar

\section{REFERÊNCIAS}

1. Department of Economic and Social Affairs, Population Division. World urbanization prospects: the 2005 revision. New York: United Nations; 2006.

2. Organização Mundial de Saúde. Guia Global: Cidade Amiga do Idoso; 2008.

3. World Health Organization. Active aging: a policy framework. Madrid: WHO; 2002.

4. Pául C. Envelhecimento activo e redes de suporte social. Porto: UNIFAI/ICBAS-UP; 2005.

5. Torres M, Marques E. Envelhecimento activo: um olhar multidimensional sobre a promoção da saúde. Estudo de caso em Viana do Castelo. VI Congresso Português de Sociologia. Mundos Sociais: saberes e práticas; Lisboa: Universidade Nova de Lisboa; 25-28 jun 2008. Número de série 233.

6. Gilroy R, Castle A. Planning for our own tomorrow. United kingdom: University of Newcastle; 2003. Electronic Working Paper n. 16.

7. Wilson G. Models of Ageing and their relation to policy formation and service provision. Policy \& Politics 1991;19(1):37-48. na formulação de políticas promotoras da mobilidade de pedestres, designadamente para as pessoas idosas (e, por isso, necessariamente para a população em geral).

Tendo por base uma visão mais abrangente do que se entende por mobilidade dos pedestres e dos fatores que a condicionam e promovem, o quadro analítico aqui apresentado procura dar um passo nesse sentido, sintetizando e definindo os principais critérios a adotar e as respectivas dimensões-chave a considerar. A adoção de uma visão conjunta desses critérios produzirá efeitos não só na qualidade do espaço público, mas também na qualidade de vida das pessoas, em particular das pessoas idosas. Mas este é um primeiro passo. O aprofundamento e a operacionalização de tais critérios exigem a mobilização de atores individuais e coletivos, sua integração neste objetivo comum com linhas de rumo estrategicamente partilhadas e a criação de compromissos conjuntos nesta missão de repensar o fenômeno do envelhecimento na agenda política das cidades.
8. Alley D, Liebig P, Pynoos J, Banerjee T, Choi IH. Creating elder-friendly communities: preparations for an aging society. J Gerontol Soc Work 2007;49(1-2):1-18.

9. Plouffe L, Kalache A. Towards global age-friendly cities: determining urban features that promote active aging. J Urban Health 2010;87(5):733-9.

10. Centeio H, Dias S, Rito S, Santinha G, Vicente H, Sousa L. Aveiro: cidade amiga das pessoas idosas!? Rev Bras Geriatr Gerontol 2010;13(3):369-81.

11. Chi-Wai L, Everingham JA, Warburton J, Cuthill M, Bartlett $\mathrm{H}$. What makes a community age-friendly: a review of international literature. Australasian Journal Ageing 2009;28(3):116-21.

12. Beard JR, Petitot C. Ageing and Urbanization: Can Cities be Designed to Foster Active Ageing? Public Health Reviews 2010;32(2):427-50.

13. Carstens DY. Site planning and design for the elderly: Issues, Guidelines, and Alternatives. New Jersey: John Wiley and Sons; 1993.

14. Tolley R, editor. Sustainable transport: planning for walking and cycling in urban environments. Cambridge, England: Woodhead Publishing; 2003. 
15. Burton, E, Mitchell L, organizadores. Inclusive urban design: streets for life. London: Architectural Press; Great Britain; 2006.

16. Daatland SO, Biggs SJ, organizadores. Aging and Diversity: multiple pathways an. Bristol, England: Policy Press; 2006.

17. Southworth M. Designing the walkable city. J Urban Plann Dev 2005;131(4):246-57.

18. Kashef M. Neighborhood design and walkability: a synthesis from planning, design, transportation and environmental health fields. AHU J Engineering \& Applied Sciences 2009;3(1):87-105.

19. Teles P, Silva P, organizadores. Desenho urbano e mobilidade para todos. Aveiro, Portugal: APPLA; 2006.

20. Ewing R, Handy S. Measuring the Unmeasurable: Urban Design Qualities Related to Walkability. J Urban Design 2009;14(1):65-84.
21. Wahl HW, Lang FR. Aging in the context across the adult life course: integrating physical and social environmental research perspectives. Annual Review Gerontol Geriatr 2004;23:1-33.

22. Mayor of London; Transport for London. Making London a walkable city: the walking plan for London; 2004.

23. Day R. Local environments and older people's health: dimensions from a comparative qualitative study in Scotland. Health Place 2008;14(2):299-312.

24. Blomley N, organizador. Rights of passage: sidewalks and the regulation of public flow. New York: Routledge; 2011

25. Koohsari MJ, Kaczynski AT. Public open space and walking: the role of proximity, perceptual qualities of the surrounding built environment, and street configuration. Environment \& Behavior [periódico na Internet]. 2012 [acesso em 24 jan 2012] 2012; Disponível em: http://eab.sagepub.com/content/ early/2012/05/04/0013916512440876.full.pdf 